\section{The Different Hues of Lipid Rafts}

\section{Gerrit van Meer}

$\mathbf{T}$ he discovery that lipid microdomains in the plasma membrane of cells sequester signaling proteins has generated much excitement. When they bind to their ligands, certain receptors (such as those expressed by $\mathrm{T}$ and $\mathrm{B}$ cells) move into lipid microdomains in the outer leaflet of the plasma membrane lipid bilayer. These domains are coupled to microdomains in the inner leaflet that contain signaling kinases, which become activated and initiate signaling cascades (1).

Specialized lipid microdomains (also called lipid rafts) in the plasma membrane outer leaflet are principally composed of sphingolipids and cholesterol. Because sphingolipids contain longer and more saturated lipid tails, the sphingolipid/cholesterol lipid rafts are thicker and less fluid than the rest of the membrane environment composed of glycerophospholipids (2). Studies of signaling pathways emanating from these lipid rafts reveal the existence of similar rafts in the inner leaflet (cytosolic surface) of the plasma membrane (see the figure). The lipid composition of cytosolic rafts is unknown, apart from their need for cholesterol to maintain their integrity and the absence of sphingolipids from the inner leaflet. The only type of lipid rafts so far identified on both sides of the plasma membrane are the caveolae, flask-shaped invaginations of the plasma membrane that are coated with the protein caveolin.

What determines the segregation of proteins into different lipid microdomains? Work by Zacharias et al. (3), reported on page 913 of this issue, provides some answers. These investigators created two variants of green fluorescent protein: one fluorescing blue under the microscope, and the other green. Neither variant was able to form dimers. By linking each variant to peptides containing consensus sequences for different lipids, the authors were able to obtain test proteins attached to different lipid anchors. They then measured the proximity (in nanometers) of the lipidmodified test proteins to different lipid mi-

The author is in the Department of Membrane Enzymology, CBLE, Institute of Biomembranes, Utrecht University, Padualaan 8, $3584 \mathrm{CH}$ Utrecht, the Netherlands. E-mail: g.vanmeer@chem.uu.nl crodomains in the plasma membrane bilayer of living cultured cells using fluorescence resonance energy transfer (FRET). Test proteins containing two lipid acyl chains (myristoyl and palmitoyl) became localized in caveolae. In contrast, test proteins containing a prenyl (geranylgeranyl) tail were found clustered together, but not in caveolae (see the figure). Thus, the test proteins were directed to different lipid microenvironments within the plasma membrane of living cells according to the type of lipid anchor that they carried.

When the temperature was lowered, the investigators were able to recover caveolin and the acylated test proteins, but not the prenylated proteins, in detergent-insoluble membrane fractions in line with earlier work (2). Traditionally, insolubility in detergent implied that the membrane proteins were associated with a cytoskeletal protein pellet. Later, investigators discovered that membranes containing sphingolipids and another class of lipid raft markers - proteins anchored to the plasma

membrane outer leaflet by a glycosylphosphatidylinositol (GPI) lipid anchor-could be "floated off" this pellet. Lipid rafts can thus be isolated (and their components identified) by floating plasma membrane preparations in detergent (2). This method has also enabled signaling proteins to be tracked as they move in and out of lipid rafts (1). However, this approach cannot discriminate between different types of lipid microdomains, for example, those containing the sphingolipid GM1 and caveolin, and those containing GPI-proteins $(4,5)$.

The addition of detergent results in the coalescence of lipid rafts that are tens of nanometers in diameter into structures that cover half of the cell surface (6). The typical lipids found in plasma membrane rafts are the sphingolipids that reside in the outer leaflet. Thus, it is unclear what fraction of the lipids in the inner leaflet form rafts in living cells and how treatment of plasma membrane preparations with detergent affects raft asymmetry. The beauty of the Zacharias et al. (3) approach is that it allows the characterization of lipid rafts without the use of detergent.

Cholesterol depletion of plasma membranes has been used successfully to identify the protein components of rafts or to monitor raft-mediated signaling, especially in the inner leaflet $(1,2,5)$. However, it should be noted that lipid microdomains can persist in the absence of cholesterol

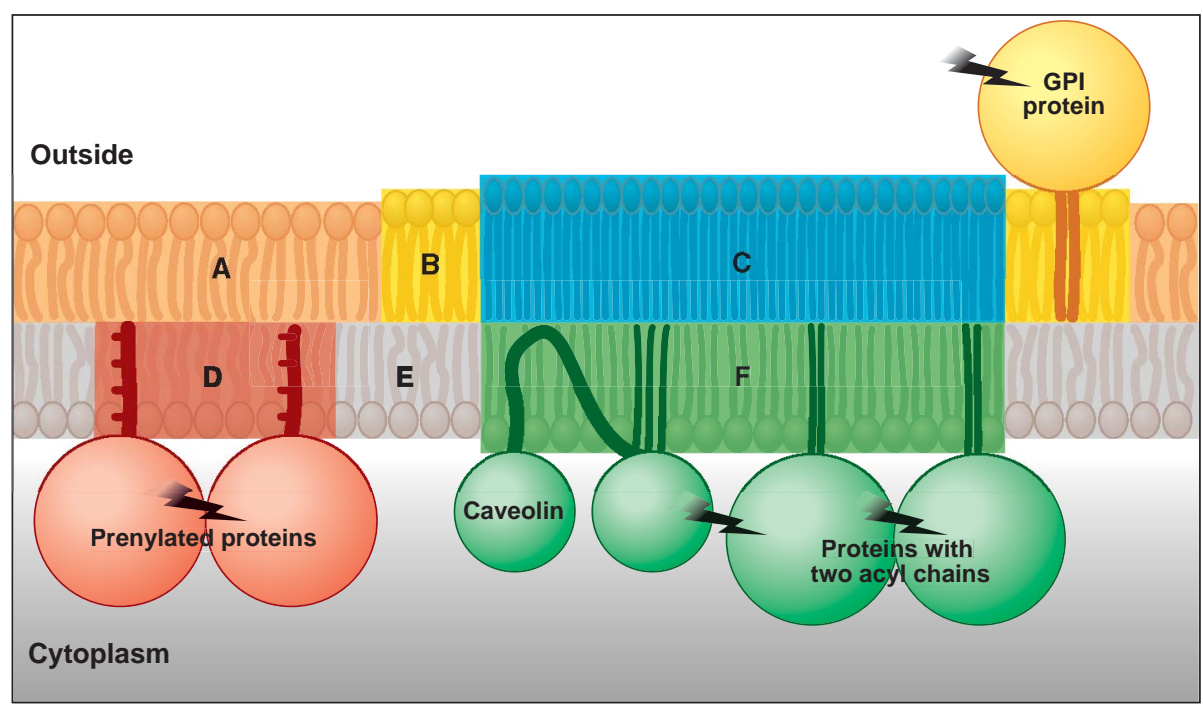

Lipid microdomains in the plasma membrane. In an environment of unsaturated glycerophospholipids (A), sphingolipids and cholesterol form less fluid microdomains (B) called lipid rafts, which are enriched for GPI-proteins. Within these microdomains, there may be even more rigid subdomains (C) enriched for the sphingolipid ganglioside GM1 (4). The inner leaflet of the plasma membrane (cytosolic surface) contains microdomains (D) of unknown lipid composition that are enriched—relative to the rest of the inner leaflet environment $(E)$ - for proteins carrying a prenyl lipid anchor. In contrast, caveolin and proteins carrying two saturated fatty acyl chains become concentrated in caveolae $(\mathbf{F})(2,3)$. Lightning bolts indicate the presence of proteins in specific lipid microdomains as revealed by fluorescence resonance energy transfer. Within a specific lipid microdomain (F), acylated proteins may be segregated into subdomains (12). 


\section{SCIENCE'S COMPASS}

and that high cholesterol levels may dissolve rafts (7). Zacharias et al. found that clustering of prenylated test proteins was insensitive to cholesterol depletion, probably indicating that these proteins clustered in a type of lipid microdomain that did not depend on cholesterol. The ability to extract prenylated test proteins with detergent (and the irregular structure of the prenyl tail) suggested that the lipid microdomains in which they congregated contained highly disordered lipids. Interestingly, replacing the saturated fatty acids bound to a signaling kinase with polyunsaturated fatty acids reduced the association of the kinase with lipid rafts, perhaps resulting in modulation of a signaling pathway (8). By using fluorescent test proteins that do not dimerize, the authors were able to avoid the pitfalls of crosslinking, which induces movement of proteins into less fluid membrane microdomains (2).
The new work still leaves many questions unanswered. What are the physical characteristics of lipid rafts in the inner leaflet, and which lipids do they contain? Why are lipid rafts in the plasma membrane outer leaflet observed under some conditions but not others $(7,9)$ ? How do proteins recognize appropriate lipid rafts, and are the increased thickness and decreased fluidity of these rafts involved? Do raft transmembrane adaptor proteins that contain palmitoyl groups, such as LAT and PAG (1), couple lipid microdomains in the outer leaflet with those in the inner leaflet? How is the actin cytoskeleton involved in raft migration and coalescence? Answers should arrive with the next wave of exciting experiments, especially those performed on cells of the immune system. Biophysicists - who have revolutionized the field by applying techniques such as single-particle tracking, single-fluorescent molecule microscopy, and atomic force microscopy-will be able to thoroughly characterize the different types of lipid rafts. Finally, it should not be forgotten that the notion of lipid rafts was developed by cell biologists studying intracellular lipid transport, a research area that will no doubt provide many more surprises $(10,11)$.

\section{References}

1. S. K. Pierce, Nature Rev. Immunol. 2, 96 (2002).

2. D. A. Brown, E. London, J. Biol. Chem. 275, 17221 (2000).

3. D. A. Zacharias, J. D. Violin, A. C. Newton, R. Y. Tsien, Science 296, 913 (2002)

4. J. E. Schnitzer et al., Science 269, 1435 (1995)

5. C. Dietrich et al., Proc. Natl. Acad. Sci. U.S.A. 98 10642 (2001).

6. M. Hao, S. Mukherjee, F. R. Maxfield, Proc. Natl. Acad. Sci. U.S.A. 98, 13072 (2001).

7. P. E. Milhiet, M. C. Giocondi, C. Le Grimellec, J. Biol. Chem. 277, 875 (2002).

8. X. Liang et al., J. Biol. Chem. 276, 30987 (2001).

9. A. K. Kenworthy, N. Petranova, M. Edidin, Mol. Biol. Cell 11, 1645 (2000)

10. V. Puri et al., J. Cell Biol. 154, 535 (2001)

11. M. Zhang et al., J. Biol. Chem. 276, 3417 (2001)

12. J. B. McCabe, L. G. Berthiaume, Mol. Biol. Cell 12, 3601 (2001).

\section{Fats, Flies, and Palmitate}

\section{Axel Nohturfft and Richard Losick}

$\mathbf{T}$ he membranes of cells are composed of a bewildering array of lipids. Yet despite their complexity, biological membranes maintain stable compositions that are characteristic for different organisms, tissues, and intracellular organelles. Evidently, homeostatic mechanisms exist that maintain the concentrations of different membrane lipids at particular levels. The best understood mechanism is a feedback system in mammals in which cholesterol and fatty acids negatively regulate their own synthesis. Reporting on page 879 of this issue, Dobrosotskaya, Seegmiller, and colleagues (I) present surprising findings demonstrating that the fruit fly Drosophila melanogaster has an identical system, but one that is regulated by phosphatidylethanolamine rather than by cholesterol or fatty acids.

More commonly known as fats, lipids are biological compounds that are soluble in organic solvents but only sparingly so in water. Lipids found in membranes have both water-soluble and water-insoluble moieties. It is this common amphipathic feature that enables the formation of the lipid bilayer, the foundation of most membranes in nature. Two abundant types of membrane

The authors are in the Department of Molecular and Cellular Biology, Harvard University, Cambridge, MA 02138, USA. E-mail: losick@mcb.harvard.edu, axno@mcb.harvard.edu lipids, phosphoglycerides and sphingolipids, are rooted in the membrane bilayer by virtue of their hydrophobic tails, which are derived from fatty acids (long hydrocarbon chains with terminal carboxylates). These molecules also carry one of several different polar head groups that can interact with water. Cells generate a staggering diversity of lipids by combining fatty acid tails (which vary in the number of carbon atoms and in the positions of double bonds) with one of several different polar head groups. Another important class of lipids is sterols, which contain a series of fused rings. One such sterol, cholesterol, is a major component of the membranes of many animal cells.

In mammals, the transcription of several crucial genes required for lipid synthesis is activated by a small family of transcription factors called SREBPs (sterol response element binding proteins). A remarkable feature of SREBPs is that their entry into the nucleus depends on their release from the membrane by proteolysis (see the figure). During their synthesis, SREBPs are inserted into the membrane of the endoplasmic reticulum, a membranous network in the cytoplasm of the cell. In the endoplasmic reticulum, SREBPs form a complex with a membrane-embedded protein called SCAP, which escorts the SREBPs to another cellular compartment called the Golgi apparatus. Here, the SREBPs are sequentially cleaved by two Golgi-specific proteases (see the figure), releasing a soluble fragment from the amino terminus. This fragment is a transcription factor, which, as a result of cleavage, is free to migrate to the nucleus, where it activates the expression of genes involved in the synthesis of cholesterol and fatty acids. Homeostasis is achieved by a negative feedback loop in which cholesterol and fatty acids block the proteolytic release of SREBPs from Golgi membranes. Interestingly, one of the SREBPs (SREBP-1c) is subject to an additional regulatory step that takes place at the promoter for SREBP-1c itself. Fatty acids, the end products of the SREBP-1c pathway, inhibit the action of a transcription factor called LXR, which is required for optimal expression of the SREBP- $1 c$ gene $(2,3)$.

How do lipids inhibit the proteolytic processing of SREBPs? This is best understood for cholesterol, which regulates the export of the SCAP-SREBP complexes from the endoplasmic reticulum. When cholesterol levels are low, SCAP escorts SREBPs to the Golgi, where processing takes place. When cholesterol levels are high, SCAP retains SREBP in the endoplasmic reticulum, processing is prevented, and cholesterol synthesis is curtailed (4).

Highlighting the central importance of the SREBP pathway for lipid homeostasis in animals, a similar cast of protein characters exists in Drosophila. These include a SREBP (flies only have one), a SCAP, and orthologs of the two proteases that release SREBP from the membrane. The SREBP pathway in flies directs the expression of genes involved in fatty acid synthesis as well as genes involved in the production of at least one membrane lipid, phos- 